\title{
Perfil epidemiológico da COVID-19 no Estado de Rondônia
}

\author{
Epidemiological profile of COVID-19 in the State of Rondônia \\ Perfil epidemiológico de COVID-19 en el Estado de Rondônia
}

\author{
Manoela Sandri Schafer \\ ORCID: https://orcid.org/0000-0002-4116-9078 \\ Centro Universitário Uninorte, Brasil \\ E-mail: manuschafer@hotmail.com \\ Clarisse Braga de Oliveira Claros \\ ORCID: https://orcid.org/0000-0002-9246-7577 \\ Centro Universitário Uninorte, Brasil \\ E-mail: clarissebclaros@gmail.com \\ Victor Yuri Pereira Damasceno \\ ORCID: https://orcid.org/0000-0003-0885-9939 \\ Centro Universitário Uninorte, Brasil \\ E-mail: victoryuri-@hotmail.com \\ Mariana Chaves Penteado \\ ORCID: https://orcid.org/0000-0002-7127-6245 \\ Centro Universitário Uninorte, Brasil \\ E-mail: marianapenteado1@gmail.com \\ Tayna Feltrin Dourado \\ ORCID: https://orcid.org/0000-0001-7310-3391 \\ Centro Universitário Uninorte, Brasil \\ E-mail: taynafd@hotmail.com \\ Ruth Silva Lima da Costa \\ ORCID: https://orcid.org/0000-0003-1890-086X \\ Centro Universitário Uninorte, Brasil \\ E-mail: ruttylyma@gmail.com
}

\begin{abstract}
Resumo
Em curto período, após o surgimento da pandemia de COVID-19, ocorreram muitos impactos de ordem econômica, política e social em todo o mundo. Na América do Sul, um dos maiores epicentros da doença, em especial o Brasil, vem evidenciando elevadas taxas de incidência e mortalidade em estados da na região norte do país. Este estudo tem por objetivo analisar o perfil epidemiológico da COVID-19 no estado de Rondônia. Trata-se de um estudo ecológico, onde foram estudados os coeficientes de incidência, mortalidade e letalidade, com base em dados secundários fornecidos pela Secretaria de Estado da Saúde de Rondônia acerca da distribuição de casos confirmados da COVID 19 no período março de 2020 a maio de 2021. Foram registrados um total de 231,873 casos e 5,824 óbitos pela doença no período analisado. Observou-se que a taxa de incidência e mortalidade por 100 mil habitantes apresentaram tendências crescentes, com a letalidade decrescente $(\mathrm{p}<0,05)$ no início do período estudado. Foi possível delinear o comportamento da patologia no estado, demostrando o seu cenário epidemiológico e promovendo dados para subsidiar as ações dos gestores em saúde da região. Novos estudos durantes e após a pandemia devem avançar no entendimento da disseminação da doença na região estudada.
\end{abstract}

Palavras-chave: Epidemiologia; Mortalidade; Incidência; Letalidade; COVID-19.

\begin{abstract}
In a short period, after the outbreak of the COVID-19 pandemic, there were many impacts of an economic, political and social order around the world. In South America, one of the largest epicenters of the disease, especially Brazil, has shown high rates of incidence and mortality in states in the northern region of the country. This study aims to analyze the epidemiological profile of COVID-19 in the state of Rondônia. This is an ecological study, where incidence, mortality and lethality coefficients were studied, based on secondary data provided by the State Health Department of Rondônia on the distribution of confirmed cases of COVID -19 in the period March 2020 to May 2021. A total of 231,873 cases and 5,824 deaths from the disease were registered in the period analyzed. It was observed that the incidence and mortality rate per 100,000 inhabitants showed increasing trends, with decreasing lethality $(\mathrm{p}<0.05)$ at the beginning of the study period. It was possible to outline the behavior of the pathology in the state, demonstrating its epidemiological scenario and promoting data to support the actions of health managers in the region. New studies during and after the pandemic should advance the understanding of the spread of the disease in the studied region.
\end{abstract}

Keywords: Epidemiology; Mortality; Incidence; Lethality; COVID-19. 


\begin{abstract}
Resumen
En un corto período, después del estallido de la pandemia COVID-19, hubo muchos impactos de orden económico, político y social en todo el mundo. En América del Sur, uno de los mayores epicentros de la enfermedad, especialmente Brasil, ha mostrado altas tasas de incidencia y mortalidad en los estados de la región norte del país. Este estudio tiene como objetivo analizar el perfil epidemiológico de COVID-19 en el estado de Rondônia. Se trata de un estudio ecológico, donde se estudiaron los coeficientes de incidencia, mortalidad y letalidad, con base en datos secundarios proporcionados por el Departamento de Salud del Estado de Rondônia sobre la distribución de casos confirmados de COVID -19 en el período de marzo de 2020 a mayo de 2021. Un total de En el período analizado se registraron 231.873 casos y 5.824 defunciones por la enfermedad. Se observó que la tasa de incidencia y mortalidad por 100.000 habitantes mostró tendencias crecientes, con letalidad decreciente $(\mathrm{p}<0,05)$ al inicio del período de estudio. Se logró perfilar el comportamiento de la patología en el estado, demostrando su escenario epidemiológico y promoviendo datos para sustentar las acciones de los gestores de salud en la región. Nuevos estudios durante y después de la pandemia deberían ayudar a comprender la propagación de la enfermedad en la región estudiada.
\end{abstract}

Palabras clave: Epidemiología; Mortalidad; Incidencia; Letalidad; COVID-19.

\title{
1. Introdução
}

Em 2020, a Organização Mundial da Saúde (OMS), sinalizou a emergência do um surto de um novo Coronavírus na China, que logo se tornou um urgente problema de saúde pública internacional. Em um curto período, foi declarado como pandemia, se proliferando em várias regiões do mundo, com diferentes impactos. O surto da doença, afetou os sistemas de saúde, o campo político e social, com impacto notável no desenvolvimento econômico global (Shrestha, Shad, Ulvi, Khan, Karamehic-Muratovic \& Nguyen et al., 2020).

Dados apontam a ocorrência de aproximadamente 180 milhões de casos registrados em todo o mundo até o momento atual e desses, um número expressivo de casos foi notificado na América do Sul, sendo recentemente declarado como o novo epicentro da pandemia (Organização Mundial de Saúde, 2021).

No Brasil, o Ministério da Saúde, registrou cerca de 13 milhões de casos, se tornando um dos países mais afetados pela doença, mesmo com a implementação de medidas de controle da sua disseminação em todo o país, como o isolamento social e a obrigatoriedade de uso de máscaras. (Urban \& Nakada, 2021; Brasil, 2021).

Mediante a isso, e apesar da crise hospitalar e sanitária, o Sistema Único de Saúde brasileiro (SUS) garantiu o acesso ao atendimento de saúde a mais de 210 milhões de habitantes, com o objetivo de deter a expansão da doença em seu território. (Marinho, Cordeiro, Coelho \& Brandão 2021).

No entanto, apesar de todos os esforços realizados pelas equipes de saúde para prevenção e controle da doença, dados evidenciaram o registro de mais de 500 mil óbitos no país (Brasil, 2021). Sendo assim, entre os 26 estados e o Distrito federal, 20 regiões evidenciaram números expressivos de casos e óbitos por COVID - 19, e dente essas, destacam-se alguns estados da região norte como o Amazonas e Rondônia (França, Ishitani, Teixeira, Abreu, Corrêa \& Marinho et al, 2020).

Destarte, no início de 2021, com o avanço da doença e o elevado número de novos casos, houve uma sobrecarga no sistema hospitalar, na maioria das cidades brasileiras, acompanhado do aumento das taxas de ocupação de leitos gerais e de unidades de terapia intensiva, gerando (UTI), levando o sistema de saúde brasileiro ao colapso (Marquitti, Coutinho, Ferreira, Borges, Portella \& Silva et al, 2021).

Frente a isso, dados do Ministério da Saúde, evidenciaram que uma das maiores taxas de incidência de COVID-19 no Brasil estão na região Norte, sendo que o estado de Rondônia, destacou-se registrando um número de casos superior aqueles apresentados pelos outros estados da região (Brasil, 2020; Escobar, Rodriguez \& Monteiro, 2020).

Analisar os dados epidemiológicos de indicadores de saúde como a incidência, mortalidade e letalidade da COVID-19 são indispensáveis para a compreensão do padrão epidêmico que pode revelar as desigualdades regionais no acesso aos serviços especializados de saúde em regiões brasileiras vulneráveis, sendo assim, e, a realização de estudos que aprofundem a investigação e caracterização de aspectos epidemiológicos da doença podem contribuir para a compreensão do seu 
comportamento na população em diferentes locais, podendo levar a intervenções oportunas e adequadas. Nesse sentido, o objetivo deste estudo analisar o perfil epidemiológico da COVID-19 no estado de Rondônia.

\section{Metodologia}

Trata-se de um estudo ecológico, onde foram estudados os coeficientes de incidência, mortalidade e letalidade, com base em dados secundários fornecidos pela Secretaria de Estado da Saúde de Rondônia acerca da distribuição de casos confirmados da COVID -19 por município, no estado de Rondônia, no período março de 2020 a maio de 2021.

O estado de Rondônia, é localizado na região norte do Brasil, apresenta uma extensão territorial de $237.765,347$ km², densidade demográfica de 6,58 hab/km², um Índice de Desenvolvimento Humano - IDH igual a 0,690 e rendimento mensal domiciliar per capita de R \$ 1.169. Estimativas do último censo realizado em 2010 , descreve que $73,5 \%$ da população deste estado reside na zona urbana (Instituto Brasileiro de Geografia e Estatística, 2020).

Além do mais, o referido estado conta com uma população heterogênica, formado por brasileiros de outras regiões do país e descendentes de estrangeiros que vieram a este espaço territorial em busca de trabalho e oportunidades de riquezas, atuando, principalmente, na extração de látex e na construção da ferrovia Madeira Mamoré. Historicamente, essa demanda migratória ocasionou vários enfrentamentos entre indígenas e não indígenas (Santos \& Alves, 2020). Na atualidade, Rondônia enfrenta conflitos agrários que compõem a narrativa das desigualdades sociais do país, caracterizada pela luta pela terra, promovida por movimentos sociais frente à pressão do agronegócio (Silva \& Dandolini, 2018).

Foram incluídos no estudo todos os casos notificados de Covid 19, bem como todos os óbitos por municípios do estado de Rondônia, no período de março de 2020 a maio de 2021. As informações foram coletadas em junho de 2021, oriundas da Secretaria do Estado de Rondônia, que disponibilizou as informações no endereço eletrônico: https://covid19.sesau.ro.gov.br, à medida que as notificações foram ocorrendo e que cujos dados são de domínio público.

Foram incluídas no estudo, todas as notificações de casos e óbitos que se referiam a COVID-19, utilizando a Classificação Internacional das Doenças, $10^{\mathrm{a}}$ edição (CID-10), de "U07.1 COVID-19, vírus identificado", associado ao diagnóstico da doença, confirmada por testes laboratoriais e disponíveis no site da secretaria de saúde. Foram excluídos dados correspondentes as outras patologias, inseridos no site da secretaria de saúde.

Os dados coletados foram transportados para planilha do Excel e então calculadas as taxas de incidência (casos novos / população) expressas por casos novos por 100,000 habitantes; mortalidade (óbitos / população) expressas número de óbitos por 100,000 habitantes, e letalidade (óbitos totais / casos totais), expressas em porcentagem. Para o cálculo foi utilizada a estimativa da Projeção da População das Unidades da Federação por sexo e grupos de idade: 2000-2030, considerando a população residente do estado de Rondônia para o ano de 2020, um total de 1,857,992 habitantes (Departamento de Informática do SUS, 2021).

As análises de tendência dos indicadores de incidência, mortalidade e letalidade foram realizadas utilizando os métodos propostos por Antunes e Cardoso (2015). Utilizou-se o modelo de regressão de Prais-Winsten as análises. O referido método possibilita que as correções de auto correlação de primeira ordem fossem realizadas nos valores, organizados por tempo. Assim, foram estimados os valores do coeficiente angular $(\beta)$ e respectiva probabilidade (p), considerando nível de significância de intervalo de confiança de 95\% (IC 95\%).

Os resultados das taxas logarítmicas ( $\beta$ ) da regressão de Prais-Winsten permitiram estimar a variação percentual de mudança diária (Daily Percent Change - DPC), com os respectivos intervalos de confiança (IC 95\%).

Esse procedimento possibilitou determinar as taxas como crescentes, decrescentes ou estacionárias. A tendência foi considerada estacionária quando $\mathrm{p}$-valor foi maior que 0,05 ( $\mathrm{p}>0,05)$. 
Por se tratar de dados de domínio público, não houve necessidade da aprovação do referido estudo em comitê de ética em pesquisa, de acordo com a resolução 466/2012 do Conselho Nacional de Saúde.

\section{Resultados}

Foram evidenciados um total de 231,873 casos e 5,824 óbitos por COVID-19 no estado de Rondônia, no período de março de 2020 a maio de 2021. Os casos e óbitos distribuídos segundo municípios de notificação foram descritos na Figura 1.

Figura 1 - Distribuição de casos confirmados e óbitos de COVID-19 no estado de Rondônia segundo Município de notificação, no período de março de 2020 a maio de 2021.

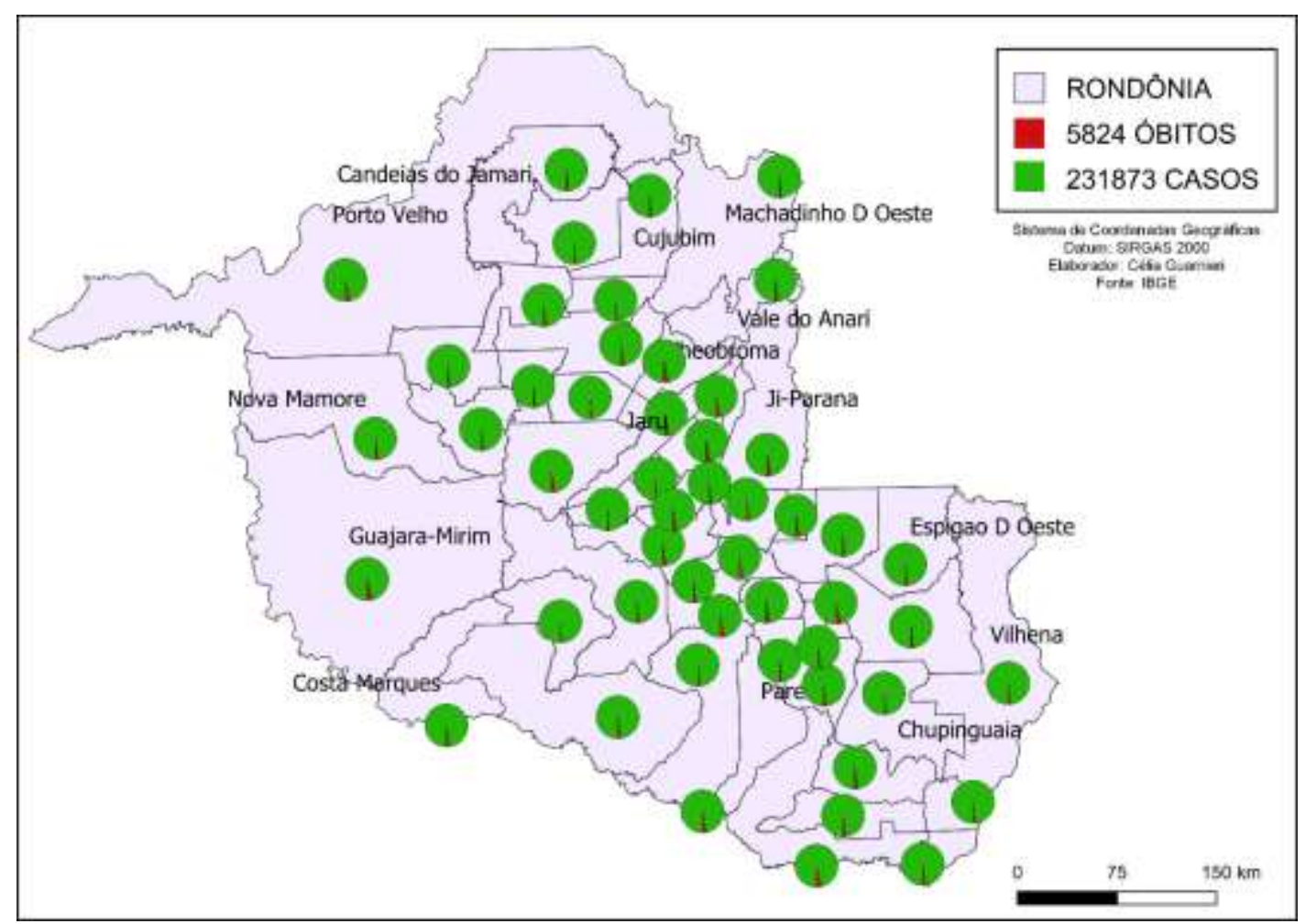

Fonte: https://covid19.sesau.ro.gov.br.

O número de casos confirmados e óbitos por COVID-19 distribuídos por mês foram ilustrados na Tabela 1 . O mês que apresentou o maior número de casos e óbitos foi março de 2021, com 39.239 (16,92\%) casos notificados da doença e $1.335(22,92 \%)$ de óbitos. 
Tabela 1 - Número de casos notificados e óbitos por COVID-19, no Estado de Rondônia, Brasil, no período de março de 2020 a maio de 2021 .

\begin{tabular}{ccccc}
\hline Mês/Ano & \multicolumn{2}{c}{ Casos } & N & Óbitos \\
\cline { 2 - 5 } & $\mathbf{N}$ & 0,01 & - & - \\
Março/2020 & 12 & 0,37 & 39 & 0,67 \\
Abril/2020 & 851 & 2,29 & 305 & 5,24 \\
Maio/2020 & 5313 & 7,83 & 437 & 7,5 \\
Junho/2020 & 18155 & 8,01 & 326 & 5,6 \\
Julho/2020 & 18573 & 6,58 & 256 & 4,4 \\
Agosto/2020 & 15254 & 4,16 & 161 & 2,76 \\
Setembro/2020 & 9638 & 2,51 & 86 & 1,48 \\
Outubro/2020 & 5821 & 4,18 & 163 & 2,8 \\
Novembro/2020 & 9691 & 6,99 & 294 & 5,05 \\
Dezembro/2020 & 16192 & 13,27 & 614 & 10,54 \\
Janeiro/2021 & 30781 & 10,4 & 781 & 13,41 \\
Fevereiro/2021 & 24113 & 16,92 & 1335 & 22,92 \\
Março/2021 & 39239 & 9,75 & 753 & 12,93 \\
Abril/2021 & 22630 & 6,73 & 274 & 4,7 \\
Maio/2021 & 15610 & $\mathbf{1 0 0 , 0 0}$ & $\mathbf{5 , 8 2 4}$ & $\mathbf{1 0 0 , 0 0}$ \\
\hline TOTAL & $\mathbf{2 3 1 . 8 7 3}$ & & & \\
\hline
\end{tabular}

Fonte: https://covid19.sesau.ro.gov.br.

As taxas de incidência da COVID-19 no estado de Rondônia, considerando o período analisado foram descritas na Figura 2. O mês de março de 2021 foi o que apresentou a maior taxa de incidência da COVID-19 com 2111,9 casos por 100 mil habitantes, seguidos pelo mês de janeiro de 2021 com 1656,68 casos por 100 mil habitantes.

As menores taxas observadas foram dos meses iniciais na pandemia com apenas 0,64 casos por 100 mil habitantes no mês de março de 2020 e 45,8 casos por 100 mil habitantes no mês de abril de 2020, enquanto que em maço de 2021 foi descrito uma taxa de incidência de 2111,90 casos por 100 mil habitantes (Figura 2). 
Figura 2 - Taxa de incidência de COVID-19, por 100 mil habitantes, no período de março de 2020 a maio de 2021 , no estado de Rondônia, Brasil.

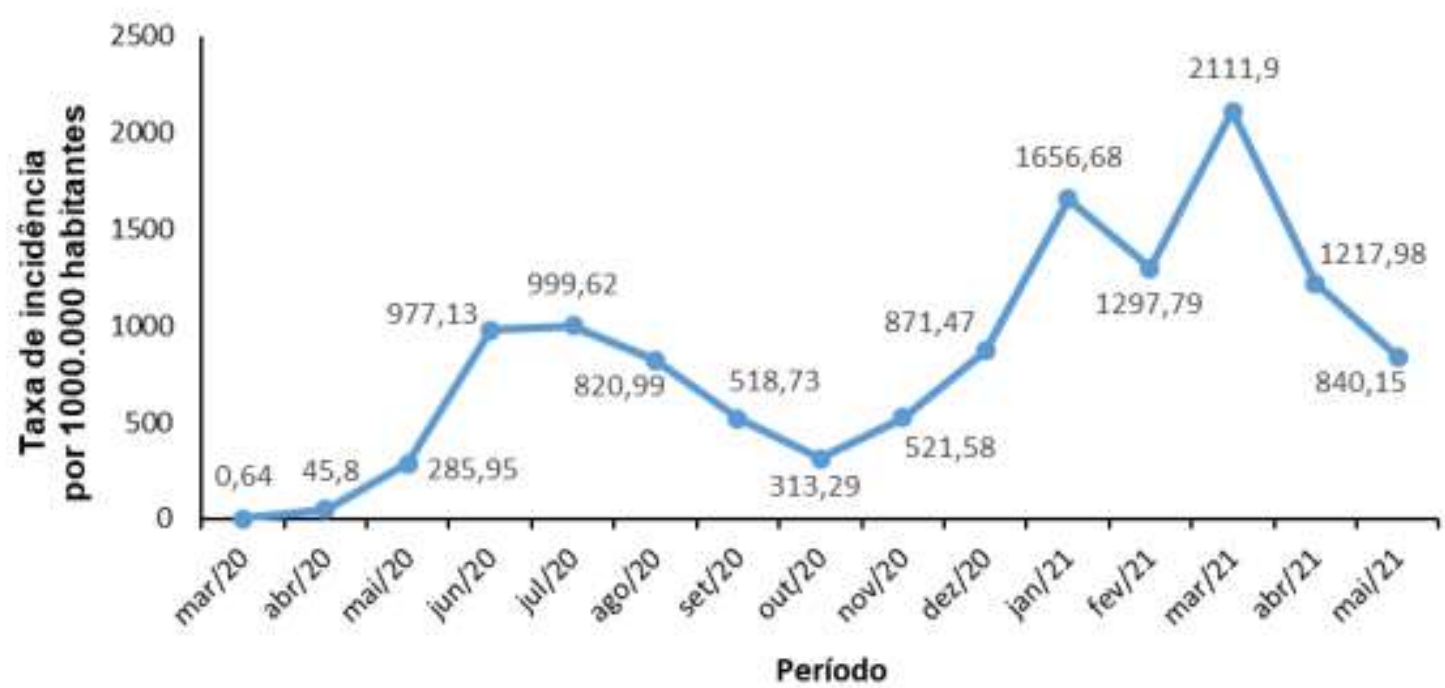

Fonte: https://covid19.sesau.ro.gov.br.

Ao analisar a taxa de incidência por 100 mil habitantes da COVID-19 no estado de Rondônia, Brasil, através das estimativas da regressão de Prais-Winsten e o Daily Percent Change, constatou-se que ela apresentou uma tendência crescente no período estudado, $\mathrm{p}<0,05$ (Tabela 2).

Tabela 2 - Estimativas da regressão de Prais-Winsten e o Daily Percent Change (DPC) das taxas de incidência por 100,000 mil habitantes da COVID-19, no estado de Rondônia, Brasil no período de março de 2020 a maio de 2021.

\begin{tabular}{cccc}
\hline Mês/ano & DPC (IC 95\%) Incidência & $\mathbf{p}$ & Tendência de Incidência \\
\hline março/2020 a maio/2021 & $0,84(0,59: 1,11)$ & $<0,001$ & Crescente \\
\hline
\end{tabular}

DPC - Daily Percent Change (\%); CI 95\% - Intervalo de Confiança 95\%; p - p valor: probabilidade de significância estatística. * Significa diferença estatística - Teste de regressão de Prais-Winsten, $\mathrm{p}<0,05$.

Fonte: https://covid19.sesau.ro.gov

As taxas de mortalidade do estado de Rondônia, Brasil, foram descritas na Figura 3. Observa-se que o pico da mortalidade ocorreu em março de 2021, com uma taxa de 71,85 casos por 100.000 habitantes, sendo que houve uma queda brusca dessa mortalidade em março de 2021 com uma taxa de 14,74 casos por 100.000 habitantes. 
Figura 3 - Taxa de mortalidade de COVID-19 (por 100 mil habitantes), no período de março de 2020 a maio de 2021 no estado de Rondônia, Brasil.

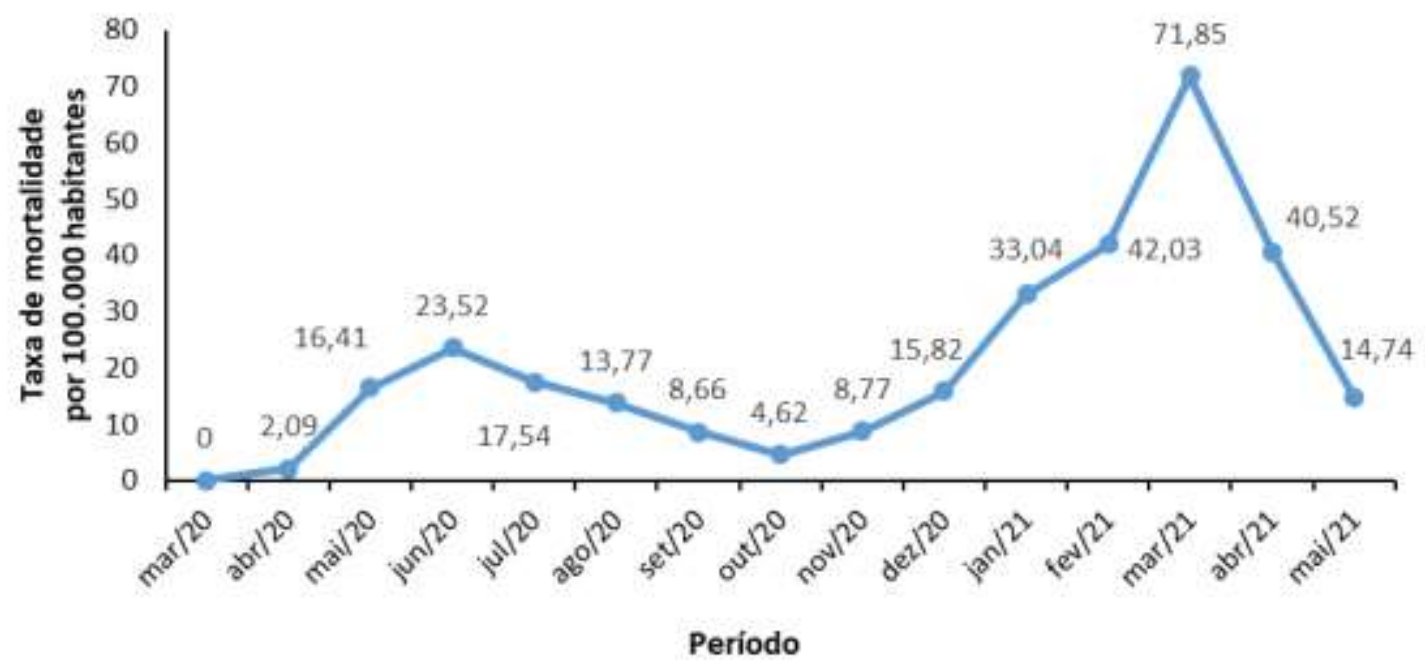

Fonte: https://covid19.sesau.ro.gov

Referente as taxas de mortalidade observadas na Figura 3, destaca-se o mês de março, onde foi evidenciado, a maior taxa, registrando 71,85 óbitos por 100,000 habitantes.

Tabela 3 - Estimativas da regressão de Prais-Winsten e o Daily Percent Change (DPC) das taxas de mortalidade (por 100 mil habitantes) da COVID-19, no estado de Rondônia, Brasil, no período de março de 2020 a março de 2021.

\begin{tabular}{cccc}
\hline Mês/ano & DPC (IC 95\%) Mortalidade & $\mathbf{p}$ & Tendência de Mortalidade \\
\hline março/2020 a maio/2021 & $0,36(0,20: 0,53)$ & $<0,001$ & Crescente \\
\hline
\end{tabular}

DPC - Daily Percent Change (\%); CI 95\% - Intervalo de Confiança 95\%; p - p valor: probabilidade de significância estatística. * Significa diferença estatística - Teste de regressão de Prais-Winsten, $\mathrm{p}<0,05$.

Fonte: https://covid19.sesau.ro.gov

A taxa de letalidade da COVID-19 no estado de Rondônia, Brasil foram descritas Figura 4. O início da pandemia no estado de Rondônia foi marcado por elevada taxa de letalidade, os maiores índices de letalidade percentual foram observados no mês de abril e maio de 2020, com os respectivos valores: 4,58\% e 5,74\%. 
Figura 4 - Letalidade percentual por COVID-19, no período de março de 2020 a maio de 2021 no estado de Rondônia, Brasil.

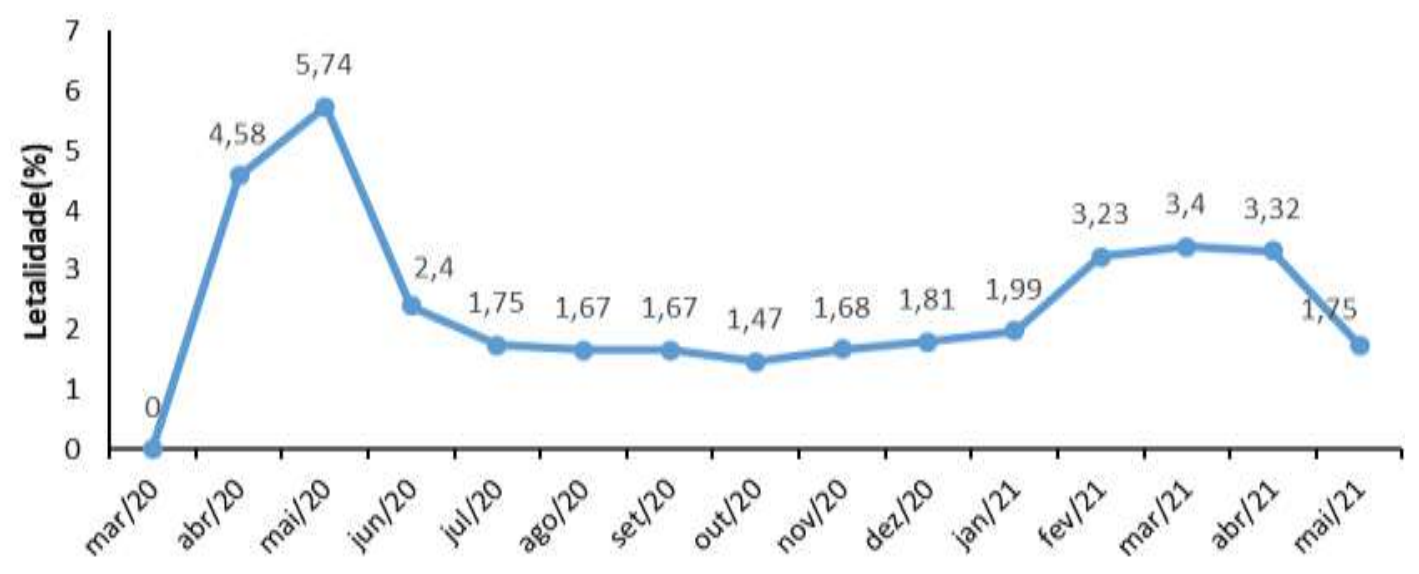

Período

Fonte: https://covid19.sesau.ro.gov.

As tendências das taxas de letalidade foram ilustradas na Tabela 4. A letalidade apresentou uma tendência decrescente, com um percentual de variação diária negativo, $(p>0,05)$, considerando o período analisado. (Tabela 4)

Tabela 4 - Estimativas da regressão de Prais-Winsten e o Dialy Percent Change (DPC) da letalidade (\%) por COVID-19, no estado de Rondônia no período de março de 2020 a maio de 2021.

\begin{tabular}{cccc}
\hline Mês/ano & DPC (IC 95\%) Letalidade & $\mathbf{p}$ & Tendência de Letalidade \\
\hline março/2020 a maio/2021 & $-0,21(-0,33:-0,09)$ & 0,001 & Decrescente \\
\hline
\end{tabular}

DPC - Daily Percent Change (\%); CI 95\% - Intervalo de Confiança 95\%; p - p valor: probabilidade de significância estatística. * Significa diferença estatística - Teste de regressão de Prais-Winsten, $\mathrm{p}<0,05$.

Fonte: https://covid19.sesau.ro.gov

\section{Discussão}

O estudo evidenciou que os casos de COVID-19 no estado de Rondônia, apresentou tendência decrescente na letalidade percentual, entretanto, destacou-se que as taxas de incidência e mortalidade por 100,000 habitantes apresentaram tendências crescentes, o que realça a importância de medidas para conter a disseminação do SARS-CoV-2 no referido estado.

A elevada taxa de incidência e mortalidade observada em março de 2021 no estado de Rondônia, também foram descritos em grande parte do Brasil. Segundo alerta emitido em boletim epidemiológico do Observatório Covid-19 da Fundação Osvaldo Cruz, onde no mesmo período foi observado uma aceleração da transmissão da doença e suas consequências sobre todo o sistema de saúde, que entrou em colapso, devido há limitados recursos hospitalares como a falta de leitos de terapia intensiva, equipamentos, medicamentos, insumos e disponibilidades de profissionais de saúde para o atendimento da demanda (Fiocruz, 2021).

Dados evidenciaram que na região norte do Brasil, concentraram-se as maiores taxas de incidência de COVID-19 no país, com 2.843,1 novos casos por 100 mil habitantes e mortalidade de 72,2 óbitos por 100 mil habitantes, sendo que o estado de Rondônia, até a $20^{\mathrm{a}}$ semana da pandemia, registrou incidência superior à apresentada pelo conjunto da região, $3.027,5$ casos por 100 mil habitantes, e mortalidade de 62,4 óbitos por 100 mil habitantes (Brasil, 2020). 
Destarte, em estudo que analisou a evolução da mortalidade pela doença no período de janeiro de 2020 a fevereiro de 2021 no Brasil, foram observadas altas taxas nos estados da região norte, apresentando um coeficiente de 174,9 mortes por 100 mil habitantes, seguida pela região Centro-Oeste $(150,9)$, Sudeste $(128,8)$, Sul $(99,5)$ e a mais baixa no Nordeste $(95,9)$, por 100 mil habitantes, respectivamente As cinco Unidades Federativas com as maiores taxas por 100 mil habitantes foram: Roraima (354,0), Amazonas (298,1), Acre $(218,7)$, Amapá $(218,6)$, e Rondônia $(213,5)$, todas da região Norte (Escobar, Rodriguez \& Monteiro, 2020).

No que tange os dados de letalidade, ao final do período analisado (maio de 2021), as taxas apresentaram índices de queda, representando um total de 1,75\% dos casos. Realça-se que em todo o Brasil a letalidade da COVID-19 vem apresentando uma redução gradual, entretanto, os referidos índices ainda estão além do esperado que é a não ocorrência de casos (Fiocruz, 2021b).

O forte impacto da pandemia, na região estudada, pode estar relacionado aos fatores sociodemográficos da população local. Segundo dados da Pesquisa Nacional de Saúde, há grandes desigualdades de acesso aos serviços de saúde entre as regiões no Brasil, com maiores proporções de consultas médicas nas regiões que apresentam as melhores condições de vida e os maiores Índices de Desenvolvimento Humano (Malta, Moura \& Bernal, 2015). Os estados da região Norte são os menos desenvolvidos (Instituto de Pesquisa Econômica Aplicada, 2016), o que pode ter contribuído para as elevadas taxas de morbimortalidade na região, pela patologia em questão.

Ainda nesse contexto, sabe-se que Brasil em 2018, 13,5 milhões de brasileiros viviam em situação de extrema pobreza, sendo assim, o cenário da região norte do país é ainda mais alarmante, posto que todos os seus estados apresentaram indicadores de pobreza acima da média nacional, o que pode gerar dificuldades quanto ao acesso aos serviços de saúde e más condições de vida e saúde da população. (Instituto Brasileiro de Geografia e Estatística, 2018; Mendonça, Rocha, Pinheiro, \& de Oliveira, 2020).

Nesse sentido, os altos índices da COVID-19 no Brasil, podem ser justificados pela grande dimensão territorial, o sistema de transporte precário que dificulta a acessibilidade dos usuários ao sistema de saúde, pelo déficit ao acesso ao saneamento básico, dificuldade de manter isolamento social sem perda importante da renda ou do trabalho. Além disso, a região Norte possui um baixo número de leitos de Unidades de Terapia Intensiva (UTI), de profissionais de saúde e equipamentos, como respiradores, os quais são amplamente necessários no tratamento e no combate das complicações desencadeadas pelas infecções por COVID-19, como é o caso do estado de Rondônia (Mendonça, Rocha, Pinheiro, \& de Oliveira, 2020; Barbosa, Galvão, Souza, Gomes, Medeiros \& Lima, 2020; Pires, Carvalho \& Xavier 2020).

Realça-se que governo do estado de Rondônia, instituiu o protocolo de medidas de higienização e prevenção contra o vírus SARS-CoV-2, que visava a promoção do distanciamento social, fechamento de escolas, comércios não essenciais e instituições públicas e privadas, além do aprimoramento dos protocolos de testagem, bem como a obrigatoriedade do uso de máscaras, dentre outras medidas, na tentativa de conter a disseminação da doença em suas regiões (Governo do estado de Rondônia, 2021a; Governo do estado de Rondônia 2021b). Entretanto, as tendências crescentes de incidência e mortalidade no atual estudo, demostram que a princípio, principalmente no início do período estudado, essas medidas não foram capazes de conter o avanço da COVID-19 na região.

Sendo assim, o presente estudo apresentou como limitações, a questão da utilização de dados secundários, que são passíveis de sofrer efeito e modificações, bem como, ajustes para viés de informações. Novos estudos durante e após a pandemia devem avançar no entendimento da disseminação da doença na região estudada. 


\section{Conclusão}

A pandemia da COVID-19 produziu impactos importantes na população do estado de Rondônia, em todas as suas regiões, com elevados números de casos notificados pela doença, bem como inúmeros óbitos.

Foi possível delinear o comportamento da patologia no estado, demostrando o seu cenário epidemiológico e promovendo dados para subsidiar as ações dos gestores em saúde da região.

Deste modo, para trabalhos futuros, sugere se a realização de novos trabalhos que sejam capazes de avaliar as medidas de prevenção e controle que estão sendo desenvolvidas junto a população, afim de medir a eficácia e eficiência das mesmas para o controle da doença.

\section{Referências}

Antunes, J. L. F., \& Cardoso, M. R. A. (2015). Uso da análise de séries temporais em estudos epidemiológicos. Epidemiologia e Serviços de Saúde, 24, 565576.

Barbosa, I. R., Galvão, M. H. R., Souza, T. A. D., Gomes, S. M., Medeiros, A. D. A., \& Lima, K. C. D. (2020). Incidence of and mortality from COVID-19 in the older Brazilian population and its relationship with contextual indicators: an ecological study. Revista Brasileira de Geriatria e Gerontologia, 23(1): e200171.

Datasus, 2021. Projeção da População das Unidades da Federação por sexo e grupos de idade: 2000-2030. <http://tabnet.datasus.gov.br/cgi/tabcgi.exe?ibge/cnv/projpopuf.def>

Escobar, A. L. (2020). A interiorização da pandemia: potenciais impactos em populações em situação de vulnerabilidade na Amazônia. NAU Social, 11(20),

Escobar, A. L., Rodriguez, T. D. M., \& Monteiro, J. C. (2020). Letalidade e características dos óbitos por COVID-19 em Rondônia: estudo observacional. Epidemiologia e Serviços de Saúde, 30.

Fiocruz. Observatório COVID-19 Boletim $\quad$ Epidemiológico. $\quad$ Semanas 11 epidemiológicas <https://agencia.fiocruz.br/sites/agencia.fiocruz.br/files/u34/boletim_covid_2021-semanas_10-11-red.pdf>

França, E. B., Ishitani, L. H., Teixeira, R. A., Abreu, D. M. X. D., Corrêa, P. R. L., Marinho, F., \& Vasconcelos, A. M. N. (2020). Óbitos por COVID-19 no Brasil: quantos e quais estamos identificando? Revista Brasileira de Epidemiologia, 23, e200053.

Instituto Brasileiro de Geografia e Estatística - IBGE. Cidades: 2020 [Internet]. Rio de Janeiro: IBGE; 2020 [citado 2020 set 24]. https://cidades.ibge.gov.br/brasil/ro/panorama

Instituto Brasileiro de Geografia e Estatística (BR). Estudos e Pesquisas-Informação Demográfica e Socioeconômica. Síntese de Indicadores Sociais: uma análise das condições de vida da população brasileira. Rio de Janeiro: IBGE; 2018.

Instituto de Pesquisa Econômica Aplicada. IPEA Desenvolvimento humano nas macrorregiões brasileiras [Internet]. Brasília, DF: 2016. http://repositorio.ipea.gov.br/bitstream/11058/6217/1/Desenvolvimento\%20humano\%20nas\%20macrorregi\%C3\%B5es\%20brasileiras.pd

Malta, D. C., Moura, L. D., \& Bernal, R. T. I. (2015). Diferenciais dos fatores de risco de Doenças Crônicas não Transmissíveis na perspectiva de raça/cor. Ciência \& Saúde Coletiva, 20, 713-725.

Marinho, P. R. D., Cordeiro, G. M., Coelho, H. F., \& Brandão, S. C. S. (2020). Covid-19 in Brazil: A sad scenario. Cytokine \& Growth Factor Reviews..

Marquitti, F. M. D., Coutinho, R. M., Ferreira, L. S., Borges, M. E., Portella, T. P., Silva, R. L. P. D., \& Kraenkel, R. A. (2021). Brazil in the face of new SARS-CoV-2 variants: emergencies and challenges in public health. Revista Brasileira de Epidemiologia, 24.

Mendonça, F. D., Rocha, S. S., Pinheiro, D. L. P., \& de Oliveira, S. V. (2020). Região Norte do Brasil e a pandemia de COVID-19: análise socioeconômica e epidemiológica/North region of Brazil and the COVID-19 pandemic: socioeconomic and epidemiologic analysis/Región Norte de Brasil y la pandemia de COVID-19: análisis... Journal Health NPEPS, 5(1), 20-37.

Ministério da Saúde (BR). Painel coronavírus [Internet]. Brasília: Ministério da Saúde; 2020 [citado 2020 ago 28]. https://covid.saude.gov.br/

Ministério da Saúde. Coronavírus/Brasil. Acesso em: 03 de abril de 2021. https://covid.saude.gov.br/..

Ministério da Saúde. Painel coronavírus [Internet]. Brasília: Ministério da Saúde; 2020. https://covid.saude.gov.br/» https://covid.saude.gov.br/

Pires, L. N., Carvalho, L., \& Xavier, L. D. L. (2020). COVID-19 e desigualdade: a distribuição dos fatores de risco no Brasil. Experiment Findings, 21.

Rondônia G do E de. Governo de Rondônia orienta população a manter medidas preventivas contra a Covid-19 [Internet]. Governo do Estado de Rondônia. [citado 28 de junho de 2021]. http://www.rondonia.ro.gov.br/governo-de-rondonia-orienta-populacao-a-manter-medidas-preventivas-contra-a-covid-19/

Rondônia G do E de. Protocolo NTE Medidas de higienização e Prevenção a COVID-19 [Internet]. Governo do Estado de Rondônia. [citado 28 de junho de 2021]. http://www.rondonia.ro.gov.br/publicacao/protocolo-nte-medidas-de-higienizacao-e-prevencao-a-covid-19/ 
Research, Society and Development, v. 10, n. 13, e243101320918, 2021

(CC BY 4.0) | ISSN 2525-3409 | DOI: http://dx.doi.org/10.33448/rsd-v10i13.20918

Santos, J. D., \& Alves, R. A. (2020). Uma análise histórica sobre a educação escolar indígena no estado de Rondônia. EDUCA-Revista Multidisciplinar em Educação, 7(17), 212-231.

Shrestha, N., Shad, M. Y., Ulvi, O., Khan, M. H., Karamehic-Muratovic, A., Nguyen, U. S. D., \& Haque, U. (2020). The impact of COVID-19 on globalization. One Health, 11, 100180 .

Silva, R. G. D. C., \& Dandolini, G. (2018). Conflitos agrários e acesso à terra em Rondônia. Revista Direito e Práxis, 9, 461-479.

Urban, R. C., \& Nakada, L. Y. K. (2021). COVID-19 pandemic: Solid waste and environmental impacts in Brazil. Science of the Total Environment, 755, 142471.

World Health Organization - WHO. COVID-19: WHO european region operational update epi weeks 31-32 (27 July-9 August) [Internet]. Geneva: World Health Organization; 2020 [cited 2020 Aug 28]. https://www.euro.who.int/_data/assets/pdf_ file/0008/460196/COVID-19-operational-update-weeks-31- 32eng.pdfrma 